\title{
Déséquilibres territoriaux en santé en Roumanie. Quel(s) rôle(s) à jouer pour l'économie sociale et solidaire?
}

Contributions de Alexandru Dragan, Alexandru Dobrita, Despina Vasilcu, Francisc Giurgiu, Christiane Beguin, Samuel Delepine, rassemblées et mises en forme par Emmanuel Bioteau pour le Centre Ligérien d'Etudes Roumaines (au sein de la Structure Fédérative de Recherches Confluences, Maison de la Recherche Germaine Tillion à Angers) afin de rendre justice aux contributeurs dans la référence aux auteurs

\section{Emmanuel Bioteau}

\section{OpenEdition}

Édition électronique

URL : http://journals.openedition.org/rfst/503

DOI : $10.4000 /$ rfst.503

ISSN : 2492-3672

Éditeur

Espaces et SOciétés (UMR 6590)

Référence électronique

Emmanuel Bioteau, « Déséquilibres territoriaux en santé en Roumanie. Quel(s) rôle(s) à jouer pour l'économie sociale et solidaire? », Revue francophone sur la santé et les territoires [En ligne], Ateliers et séminaires, mis en ligne le 07 juin 2016, consulté le 06 avril 2021. URL : http:// journals.openedition.org/rfst/503; DOI : https://doi.org/10.4000/rfst.503

Ce document a été généré automatiquement le 6 avril 2021.

\section{(ब) (1)}

La Revue francophone sur la santé et les territoires est mise à disposition selon les termes de la Licence Creative Commons Attribution - Pas d'Utilisation Commerciale - Partage dans les Mêmes Conditions 4.0 International. 


\section{Déséquilibres territoriaux en santé en Roumanie. Quel(s) rôle(s) à jouer pour l'économie sociale et solidaire?}

Contributions de Alexandru Dragan, Alexandru Dobrita, Despina Vasilcu, Francisc Giurgiu, Christiane Beguin, Samuel Delepine, rassemblées et mises en forme par Emmanuel Bioteau pour le Centre Ligérien d'Etudes Roumaines (au sein de la Structure Fédérative de Recherches Confluences, Maison de la Recherche Germaine Tillion à Angers) afin de rendre justice aux contributeurs dans la référence aux auteurs

\section{Emmanuel Bioteau}

\section{NOTE DE L'ÉDITEUR}

Avec la collaboration de : Alexandru Dragan [contributeur], Alexandru Dobrita [contributeur], Despina Vasilcu [contributeur], Francisc Giurgiu [contributeur], Christiane Beguin [contributeur], Samuel Delepine [contributeur]

1 La séance a été convoquée à l'initiative de, et sous la présidence de, Emmanuel Bioteau. Il est proposé un tour de table préalable. Sont présents Mesdames Sylvie Mayer (Fondation Gabriel Péri, co-organisatrice du colloque des 7, 8 et 9 juin) et Christiane Béguin (Réseau OVR Solidarités Suisse), Messieurs Francisc Giurgiu (Réseau OVR Solidarités International, et OVR Solidarités Roumanie), Jacques Prinet (Réseau OVR Solidarités France, Comité de Jumelage Beaupréau-Posesti et Coordination AnjouRoumanie), Jean-Marie Dessèvre (Comité de Jumelage Beaupréau-Posesti et Coordination Anjou-Roumanie), Jean-Pierre Rolandeau (Association d'Addictologie du Maine et Loire), Samuel Delépine (Géographe, Maître de conférences, Centre Ligerién d'Etudes Roumaines et ESO-Angers), Jérôme Prugneau (Géographe, doctorant, ESO- 
Angers), Alexandru Dragan (Géographe, doctorant, ESO-Angers et Université de l'Ouest de Timisoara), Alexandru Dobrita (Géographe, étudiant en Master, ESO-Angers et Université de l'Ouest de Timisoara), Emmanuel Bioteau (Géographe, Maître de conférences, Centre Ligerién d'Etudes Roumaines et ESO-Angers). Sont excusés, entre autres: Madame Despina Vasilcu (Géographe, Université de Suceava), Messieurs Nicolae Popa (Géographe, Professeur, Université de l'Ouest de Timisoara), Sébastien Fleuret (Géographe, Directeur de Recherches du CNRS, Directeur de site d'ESO-Angers).

2 Le compte rendu de cet atelier comporte 4 parties :

3 (1) Retour sur l'historique et les réalisations de ce séminaire (en 3 rencontres).

4 (2) Etat des lieux des problématiques rencontrées en Roumanie: au travers de deux témoignages, bilans de travaux scientifiques (interventions de Alexandru Dragan et de Alexandru Dobrita).

5 (3) Cinq axes de recherches développées durant ce séminaire.

6 (4) Conclusion générale. Perspectives de valorisation et suites données au séminaire.

\section{Retour sur l'historique et les réalisations de ce séminaire}

7 Ce séminaire émane du projet "Politiques Publiques et Action Sociale » lequel envisageait la construction d'un protocole d'études associant Recherche appliquée, Construction d'un consortium franco-roumain (appelé à s'élargir ensuite au niveau européen) et Formation (en particulier des étudiants de Master inscrits à l'Université d'Angers - filières M1-M2 "Intervention Sociale» et «Chargé de Développement Entreprises et Territoires, Cultures », ce dernier étant co-porté par les UFR Lettres LSH et $\mathrm{DEG})$. Ce projet était initialement programmé pour une période allant de janvier 2015 à décembre 2016, et avait fait l'objet du dépôt d'une demande de financements auprès de la Région Pays de la Loire dans le cadre du dispositif de soutien aux travaux scientifiques en SHS du CPER 2010-2016. Après un report initial, le financement a été alloué au travers d'un dispositif régional (Pays de la Loire), appuyé par l'agglomération angevine (Angers Loire Métropole) de soutien aux coopérations interuniversitaires et aux formations, pour une période allant de juillet 2015 à juin 2016.

8 Ce séminaire s'inscrit également dans deux des axes de travail de la SFR Confluences : Axe 2: Changement social: genre, discriminations, inégalités et Axe $4:$ Innovations, Développement, Solidarités. Ces deux entrées thématiques rejoignent 3 des axes de recherche de la MSH régionale Ange Guépin (à laquelle est rattachée la Maison de la Recherche Germaine Tillion d'Angers), à savoir: "Travail, Formation, Solidarités ", "Santé et Société » et "Identités, Territoires, Cultures ».

9 Le projet vise par une entrée territoriale à identifier en Roumanie différentes situations relatives à l'offre de services sociaux, médico-sociaux et en santé. Il s'agit par cela de produire (1) une typologie des différentes situations, et l'analyse de ces situations, (2) de comprendre les effets des régulations publiques à caractères social et médical sur les territoires, leurs habitants, leurs établissements, (3) d'analyser les raisons du nonrecours à certains services et (4) de formaliser les modalités (leur nécessité réelle ?, leur applicabilité ?) d'un transfert de pratiques entre la France et la Roumanie (et inversement) tant en termes de formation que pour l'action publique et/ou civile 
locale. Il associe pour cela des acteurs institutionnels et civils en Roumanie (Directions départementales de Santé et de Protection de l'Enfance du Timis (département), Réseau OVR Solidarités (Opération Villages Roumains), ONG Fundatia de Abilitare Speranta, ONG Ajutor Maltez...) et en France (Coordination Anjou-Roumanie entre autres). Ces différents acteurs ont pu être réunis tant en juin 2015 et en juin 2016 à Angers qu'en mars 2016 à Timisoara.

10 Par ailleurs, des stages ont vu le jour, associant là encore les différentes parties prenantes de ce séminaire. Pour rappel :

- 3 étudiantes angevines (Célia Brière, Noémie Perret, Domitille Thiebot, M1 DAST) accueillies à Timisoara au printemps 2015 dans les organisations suivantes :

- Direction départementale de la Jeunesse et de la Protection de l’Enfance Timis,

- Fondation Abilitare Speranta,

- Service d'Aide Maltaise Timisoara.

- 1 étudiant (Jérémy Macé, M1 Géographie) à Angers dont le stage est en cours à l'heure de cette rencontre finale, consistant en l'identification, en France, des rapports de la population aux services de santé de proximité; les apports de ce stage peuvent être considérés au titre des perspectives de prolongement du séminaire dans des retours croisés d'expériences, au bénéfice d'apprentissages communs.

- 1 étudiant de Timisoara (Alexandru Dobrita, M2 PDDT et M2 CDET) à Angers dont le stage est en cours à l'heure de cette rencontre finale, consistant en l'identification problématiques et besoins en services de santé en Roumanie.

11 Au-delà du cycle de rencontres programmées pour ce séminaire, différents temps d'échanges ont pu être organisés à l'initiative de ces acteurs: Rencontres nationales d'OVR-France le 21 novembre 2015 à Pouancé (Maine et Loire), en présence de Emmanuel Bioteau et des trois étudiantes accueillies à Timisoara au printemps 2015 lesquelles ont pu ainsi témoigner de leur expérience; Rencontres internationales d'OVR le 16 avril 2016 à Moutiers (Suisse), en présence de Emmanuel Bioteau.

Ce projet émane de ressentis, d'intuitions, mais également de l'expérience des différents acteurs présentés auparavant, lesquels pointaient des besoins en santé et dans la mobilisation de la société civile roumaine. Au début de la décennie 2010, les bénévoles du réseau OVR constataient des difficultés à soutenir le maintien des jeunes dans les milieux ruraux roumains faute en particulier d'une offre de soins de qualité dans ces communes. Les rencontres de ces bénévoles, acteurs du développement social et solidaire du quotidien, avec les chercheurs du CLER permettaient que soient croisées les analyses des uns et des autres. Car dans le même temps, les chercheurs réunis dans le CLER (Centre Ligérien d'Etudes Roumaines) produisaient des analyses sur l'évolution du système de santé (et assurantiel en santé) roumain (FLEURET, BIOTEAU, 2011), sur les difficultés d'accès aux soins des populations les plus pauvres, parfois stigmatisées tels les Roms (DELEPINE), ou encore amorçaient des travaux sur les capacités de mobilisation de la société civile (DRAGAN). Ces travaux rejoignaient les analyses conduites sur les pratiques en santé, les migrations de médecins (VASILCU), les défauts d'animation de la société civile roumaine (PIROTTE)...

13 Au final ces différents travaux, produits en langues française, roumaine ou anglaise pointent systématiquement trois effets qui sont au cœur de notre approche, et en constituent le socle originel et l'originalité :

- L'état de santé de la population roumaine est l'un des moins satisfaisants d'Europe et

le recours aux services sociaux - le plus souvent gratuits, proposés par des ONG - est 
également faible malgré les besoins . L'espérance de vie moyenne y est, par exemple, de 74,5 ans en 2014 , soit près de six années en dessous de celle de l'Union européenne enregistrée fin 2013 (80 ans). Cette statistique démontre le déséquilibre fondamental qui persiste entre l'ouest et l'est de l'Europe. Ce déséquilibre s'est accentué plutôt que résorbé durant la décennie 1990, du moins en Roumanie: durant cette période, les indices de mortalité, en particulier de mortalité maternelle et infantile ${ }^{2}$, comme les indices de morbidité, sont en hausse (ECSHD, 2006). Ce constat motive l'effort de réorganisation de l'assurance santé et des soins, mais également de l'ensemble de l'offre de services d'intervention sociale en Roumanie. Les régions de l'ouest de la Roumanie et les grandes villes (universitaires) sont en tête des offres de services (BIOTEAU, 2005). Mais on peut aussi y voir un effet de la réforme, d'abord budgétaire, amorcée à l'occasion du changement de régime consécutif à la chute de l'Etat communiste.

- Si des formations professionnelles existent à présent (formations d'assistance sociale - un séminaire du Centre Ligérien d'Etudes Roumaine a été consacré à ce sujet le 23 janvier à Angers), dans le giron des universités et en lien avec les filières de sociologie-psychologie et/ ou de théologie, les personnels formés peinent à trouver des postes en correspondance avec leur qualification et qui soient dignement rétribués. Par conséquent, l'action sociale de proximité en Roumanie reste l'apanage des ONG et de quelques agences gouvernementales déconcentrées à l'échelle des départements. Le manque de moyens financiers, l'absence d'aides sociales d'État et l'absence de normes tarifaires, créent alors des inégalités spatiales fortes dans l'accès à ces services.

- Enfin, il n'existe pas à proprement parler un secteur médico-social. Le volet médical reste l'apanage des personnels de santé, des cliniques privées et des hôpitaux. Le secteur de l'assistance sociale renvoie principalement aux problématiques de compensation des effets de la pauvreté financière, de l'isolement (minorité Rom, personnes âgées sans ressources, enfance abandonnée ou fragilisée) et aux situations de handicap. Les incidences sur la population sont alors à rechercher du côté de l'absence de ponts entre traitement social et traitement par le soin des problématiques individuelles et collectives de santé.

\section{Témoignages}

Deux témoignages structurent cette demi-journée d'échanges à Angers. Un troisième témoignage est apporté par la communication de Despina Vasilcu à l'occasion du colloque en économie sociale et solidaire du lendemain! Il en est rendu compte durant les échanges.

Néanmoins en amont de ces derniers, Emmanuel Bioteau revient sur les principaux apports des deux précédentes sessions de séminaire. Ainsi s'impose de façon prégnante la problématique de l'émigration des personnels de soins et de santé (médecins mais également infirmiers, professions paramédicales et d'intervention sociale) (KRASTEVA et VASILCU, 2014), à laquelle s'ajoutent les tendances observées sur les jeunes en formation lesquels envisagent leur carrière à l'étranger (POPA et LUCHES, 2014). De fait, le pays se vide de ses personnels de santé. Si les données officielles font apparaître une émigration massive, celle-ci ne semble toutefois pas être une problématique majeure au plan national: la Roumanie conserve un ratio correct de personnels de santé par habitants (certes bas pour l'Europe). Mais ces données masquent des disparités zonales extrêmement marquées, menaçant certains secteurs géographiques d'une complète déprise médicale, en particulier dans les milieux ruraux. Les 
témoignages de Messieurs DRAGAN et GIURGIU sont en ce sens éloquents : chacun d'eux évoque le cas de communes dépourvues de personnels de santé. Dans le cas de la région de Lipova (judet Arad), un seul médecin généraliste officie actuellement, couvrant des villages s'étalant sur plus d'une cinquantaine de kilomètres de distance.

Ces témoignages illustrent les principales problématiques liées à la restructuration de l'organisation territoriale en santé en Roumanie (depuis la sortie du communisme), que nous pouvons lister ici :

- Changements des régimes assurantiels en santé, et défaut de confiance de la population dans le régime général actuel.

- Défaut de présence, de mobilisation de la société civile institutionnalisée; besoins d'un renforcement des initiatives communautaires.

- Poids extrêmement important des élus locaux dans la mise en œuvre d'actions phares: renforts ou entraves selon les cas.

- Poids des mouvements religieux... jouant à l'identique de celui des élus locaux.

- Des déséquilibres territoriaux (entre rural et urbain, entre l'ouest et l'est du pays, entre les principaux sites universitaires en santé et le reste du pays...), induits par l'ensemble des constats précédents. Aux risques de ruptures spatiales?

17 Il se pose alors différents enjeux pour l'avenir, qui sont autant de questions posées aux participants de ce séminaire :

- Gestion des migrations entrantes dans le pays : des personnels de santé en provenance de République de Moldavie, du Proche-Orient, mais également de nouveaux habitants (où l'on pense plus particulièrement aux réfugiés actuellement en transit vers l'Europe à travers le pays).

- Un renforcement des déséquilibres territoriaux constatés, aux risques d'une désertification médicale, et globale : l'absence de personnels de santé révélant l'absence d'attractivité pour les plus jeunes faute d'offres d'emplois, de formation, d'accès à la culture, etc. Ce qui rejoint un constat dressé par ailleurs en France, Suisse, etc. à divers degrés.

- Avec la problématique du financement de l'offre de soins et en santé, un rôle nouveau à jouer pour des initiatives solidaires.

\section{Intervention de Alexandru Dragan}

18 (Doctorant en géographie, cotutelle de thèse entre Université d'Angers, ESO-Angers (UMR 6590 CNRS) et Université de l'Ouest de Timisoara, Roumanie - thèse soutenue à l'Université d'Angers le vendredi 9 septembre 2016).

19 Les CARP (Caisses d'Aide Réciproque pour les Retraités), un modèle possible pour le secteur médico-social ? L'exemple de la CARP de Timisoara.

Alexandru Dragan s'exprime sur une thématique abordée dans le cadre de sa thèse de doctorat (soutenue depuis, en septembre 2016) portant sur L'émergence et la structuration de l'Economie sociale et solidaire en Roumanie. Au sein des enquêtes conduites dans le Banat (région de Timisoara, vallée du Jiu, petites villes de Lipova et de Jimbolia...) le domaine de la santé est cité dans seulement $11 \%$ des cas d'actions bénévoles dans lesquelles se sont engagés les répondants (sachant que pour un échantillon total de plus de 800 répondants, à peine $17 \%$ indiquent avoir effectué du bénévolat. La part d'engagement bénévole en santé est donc extrêmement limitée. Nous pourrons cependant retenir que $20 \%$ des répondants à une seconde enquête envisageraient de faire du bénévolat dans les actions de santé dans un futur proche, 
mais à condition que l'on puisse leur offrir les moyens, le cadre, de telles actions. C'est bien là ce qui semble aujourd'hui poser le plus problème, et rejoins les interrogations portant sur la structuration d'une société civile active en Roumanie. Ce qui fait défaut est le bénévolat indirect, ou d'investissement, c'est-à-dire un bénévolat d'encadrement et de programmation des actions à mener " par d'autres».

21 Ses résultats d'enquêtes permettent également de pointer un manque d'initiatives issues du niveau local dans ses terrains (zones rurales et Vallée du Jiu), alors que, à Timisoara, l'on peut identifier une grande diversité d'actions. Le public y est plus jeune, sensibilisé à des modes d'agir différents de ceux des générations précédentes. Surtout la densité d'Organisations Non Gouvernementales s'y trouve plus élevée alors que, enfin, les solidarités interpersonnelles, de proximité, encore visibles dans les campagnes et dans les petites villes (bien que fragilisées) ne jouent plus, ou moins, en milieu urbain.

L'exemple de la CARP de Timisoara est révélateur. A. DRAGAN reprend un passage de sa thèse lequel illustre cette évolution du secteur ESS en santé en Roumanie d'une part, et le rôle que pourrait jouer dans le futur des institutions du type des CARP :

«En 2015, le nombre des retraités a dépassé 5,3 millions de personnes, pour 4,4 millions de salariés. Cette population ne bénéficie pas d'une couverture médicale complète et gratuite (le système de soin dentaire est généralement libéral) et l'indemnisation moyenne ne dépasse pas 190 euros par mois ${ }^{3}$. Cette situation limite l'accès de cette catégorie à de nombreux services.

C'est dans ce contexte que la CARP répond à des besoins, parfois des urgences, nonsatisfaites par l'État ou par le marché. Dans le cas des emprunts, il suffit d'être membre depuis six mois pour avoir accès au prêt. En règle générale, il s'agit de petites sommes. Par exemple, les prêts de moins de 250 euros ont un taux d'intérêt de $7 \%$ et doivent être remboursés dans 12 mois. Plus la somme augmente, plus le taux d'intérêt augmente.

La palette des services offerts par la CARP dépasse l'aspect financier. L'objectif est désormais le « désenclavement » des bénéficiaires. La structure dispose d'un centre de loisir à $20 \mathrm{~km}$ est de Timișoara, disponible gratuitement aux membres, d'une bibliothèque, elle organise aussi des excursions en Roumanie et à l'étranger et dispose de sa propre chorale et de son atelier de peinture.

Les services les plus complexes sont dans le domaine de la santé. La polyclinique de la CARP est composée des cabinets de stomatologie, de cardiologie, de gastroentérologie, ou de kinetophysiothérapie. Les services sont offerts aux membres soit gratuitement, soit pour des prix au-dessous du marché.

Dans le choix de la promotion de ses activités, la CARP développe un partenariat avec l'Office national des pensions, qui, lors de la première retraite, distribue aux personnes des matériaux publicitaires de la CARP. Cette relation fonctionne également comme mécanisme de contrôle: dans le cas où les membres ne remboursent pas le prêt, la somme est prélevée automatiquement de son indemnisation mensuelle de retraite.

D'après les acteurs rencontrés, le siège social du CARP en soi est un outil de promotion (voir groupe photo 1). Le bâtiment est entièrement rénové et situé sur une artère principale de la ville, à la limite du quartier le plus dense de Timișoara. Un arrêt de tramway se situe à 50 mètres du bâtiment. Par le transport public, la place centrale de la ville et la gare sont accessibles en cinq minutes. Cela favorise un grand afflux de personnes, comme nous avons pu le constater à plusieurs reprises lors des visites sur place.

La « vitrine » du siège n'est pas très différente de l'entrée d'un centre médical privé. Tous les services sont affichés d'une manière détaillée et sous une forme attractive (images, couleurs dynamiques, etc.). 
Une dernière remarque concerne la capacité de la CARP à couvrir une échelle géographique plus large que la ville uniquement. En effet, toutes les autres villes du département de Timiș (sauf Lugoj, qui a sa propre caisse) ont un représentant de la structure qui collecte la cotisation mensuelle ou distribue les prêts. Mais les services disponibles à Timișoara ne sont pas directement accessibles. De plus, dans le milieu rural, il existe très peu de représentants. Cela témoigne de nouveau de la situation de précarité multiple connue par la population de ce milieu » (DRAGAN, 2016, 117-118) la nécessité de sensibiliser, depuis la France, la Suisse, et autres Etats d'Europe occidentale, les élus auprès des instances européennes : les sensibiliser à la nécessité d'un renforcement de la formation des cadres en Roumanie, et d'un soutien plus actif aux initiatives de la société civile dans ce pays comme dans les Etats voisins.

\section{Intervention de Alexandru Dobrita}

27 (Master Chargé de Développement Entreprises et Territoires durables de l'Université d'Angers et Master Planification et Développement Durable des Territoires de l'Université de l'Ouest de Timisoara, dispositif de double diplôme - stagiaire à ESOAngers (UMR 6590 CNRS) en avril, mai et juin 2016). 

précédents ateliers. La carte suivante en témoigne. Les médecins sont plus densément présents dans l'ouest que dans l'est du pays, également dans les départements (judet) abritant des universités en médecine. Les quatre départements aux plus fortes densités (Bucarest, Cluj, Timis, Mures), mais également à l'est Iasi et Constanta abritent des universités de médecine. Enfin, les départements à dominante rurale apparaissent ici en creux, marqués par de très faibles densités (cela y compris dans le cas de présence de grandes villes).

Proportion de médecins pour 1000 habitants, par judet (département) en 2012



Il rend compte d'un stage de Master portant sur La problématique d'accessibilité sociale et territoriale au système sanitaire dans le département Timis en Roumanie.

Il pointe dans ce travail les problématiques de déséquilibres spatiaux évoquées lors des

A. Dobrita pointe également une série d'autres problèmes. La plupart des médecins opérant dans les zones rurales du département Timis résident en ville.

Il met ensuite en avant différents exemples de rapports rendus difficiles dans l'articulation entre les besoins locaux (et initiatives locales) et la nécessité d'un équilibre territorial (à toutes échelles).

Ainsi, un exemple peut être donné à travers le programme de prévention des grossesses non désirées, considérant que le taux d'avortement est élevé en Roumanie, plus particulièrement en milieu rural. Ce programme porté par la Direction de la Santé Publique du Timis coordonne huit cabinets en charge du planning familial. Ces cabinets sont prioritairement destinés aux publics étudiants, aux personnes résidant en milieu rural, aux personnes bénéficiaires de l'aide sociale. Mais ces cabinets « labellisés », et de ce faits soutenus financièrement, sont présents en milieux urbains uniquement, dans les villes de Timisoara (plus de 300.000 habitants), Lugoj (plus de 50.000 habitants), Jimbolia, Deta, Sânnicolau Mare (toutes plus de 10.000 habitants) ${ }^{4}$. Cet exemple montre l'écart entre les objectifs des politiques publiques en santé et leurs applications territoriales. Et pourtant le département du Timis reste l'un des mieux doté en la 
matière. Au final, les campagnes ne sont quasiment pas couvertes par ces dispositifs de prévention en santé.

Autre exemple : des communes de plus de 5.000 habitants ont sollicité des autorités départementales ( $\mathrm{du}$ Timis toujours) la possibilité d'ouverture d'un centre de soins permanent, abritant personnels médicaux (médecins, infirmiers) et paramédicaux. Ce dispositif est permis légalement mais il est soumis à une cartographie précise; dans le cas du Timis, ces communes sont jugées comme étant trop proches de centres hospitaliers (dans un rayon de moins de $30 \mathrm{Km}$ ), d'une part, et la densité de services de santé est jugée (ce que confirme la carte) comme étant déjà élevée au regard du reste du pays. Ces initiatives municipales ne peuvent donc pas recevoir le soutien technique et financier des autorités départementales (rappelons qu'en Roumanie, le département - ou judet - agit comme entité déconcentrée, et non pas décentralisée, dans le domaine de la santé).

Dans ce contexte, certaines communes restent, malgré leur poids démographique, sans médecins.

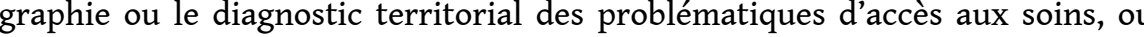
encore de données de santé ou de présence des personnels médicaux, fait état d'une situation posant problèmes à échelles macro ou méso: par comparaisons entre la Roumanie et ses voisins, mais également à l'intérieur du pays...

Ces projections sont réalisées de façon à assurer un équilibre interne au pays, entre départements. Mais ces données et leurs exploitations sont, en conclusion, trompeuses. Elles tendent à gommer les écarts à échelle micro, qui se maintiennent aux échelons communaux voire entre quartiers, ou entre villages dans les campagnes. Ces écarts sont d'autant plus problématiques que, n'étant pas rendus visibles par la plupart des projections, ils ne sont pas pris en compte dans les politiques publiques. Au final, l'écart entre villes et campagnes est grandissant, ce qui tend à renforcer le rejet des campagnes des jeunes générations.

La problématique est ainsi double et semble irrésoluble en l'état: le sous équipement des campagnes ne pourra pas trouver réponse dans des politiques étatiques visant à l'équilibre entre départements d'une part; le sous équipement des campagnes pousse les jeunes générations à s'installer dans les principales villes du pays, renforçant ainsi le déséquilibre entre les départements dotés de ces grandes villes et les autres.

Les représentants du réseau OVR Solidarités témoignent en ce sens. Monsieur Giurgiu notamment rappelle combien il peut être difficile aujourd'hui de structurer une offre médicale dans les communes rurales. Il souligne également à travers l'exemple de communes de Moldavie roumaine, le danger qu'il y a à voir des médecins âgés non remplacés dans les petites villes. En cela les deux communications de Messieurs Dragan et Dobrita se rejoignent.

Alexandru Dragan remobilise également à ce moment l'exemple travaillé par des étudiantes angevines en stage à Timisoara courant 2015 : elles étaient présentes dans une fondation (Abilitare Speranta) laquelle propose des soins pour des enfants souffrant de handicaps variés. Ces soins très spécialisés sont également assurés par quelques autres fondations, mais toutes sont présentes dans la ville de Timisoara. Aucune autre offre n'est disponible à l'échelle du sud-ouest de la Roumanie. Les responsables de la fondation témoignaient alors d'exemples de parents qui, lassés des 
allers-retours incessants sur plusieurs centaines de kilomètres, avaient fait le choix de quitter leur résidence d'origine pour Timisoara.

Ce cas n'est pas isolé en Roumanie. Il n'est pas non plus singulier à la Roumanie. Nous rappellerons qu'en France par exemple, et en particulier dans le cadre du handicap, des situations familiales analogues peuvent être identifiées. Cependant dans le contexte roumain, cet écart entre l'offre et la demande en santé est quasi exclusivement à l'avantage des principaux centres urbains; jamais voire rarement de petites villes pourront jouer ce rôle attractif; au contraire même, on les quittera pour la grande ville proche.

\section{Apports des travaux de Despina Vasilcu sur les Migrations des médecins roumains : enjeux, tendances et impacts}

41 Si l'on dresse un état des lieux de la migration des médecins roumains vers l'Europe, nous pouvons identifier une dynamique sans précédent de la migration des professionnels de santé roumains, suite à la reconnaissance, depuis 1 er janvier 2007 date de l'adhésion de la Roumanie à l'Union européenne - du diplôme en médecine obtenu en Roumanie. Cela instaure une nouvelle étape dans le paysage migratoire européen induite par la libre circulation, d'installation et de travail. Ce faisant, le flux initial déjà élevé des décennies 1990 et 2000 s'accroît de façon spectaculaire. En particulier en direction de France : les médecins de nationalité romaine détenaient, au début de l'année 2013, la première place parmi les médecins à diplôme européen $(36,4 \%)$ qui exerçaient leur profession en France, suivi par les Belges (21\%), les Italiens (11\%), et les Allemands (10,1\%). Dans l'absolu cependant, l'Allemagne (en raison des migrations de citoyens roumains de nationalité allemande, ce dès les années 1960, phénomène accéléré dans les années 1990) et le Royaume-Uni, constituent les premières destinations européennes devant la France pour les médecins roumains. La migration des médecins roumains comble le déficit de personnel qualifié dans certains pays européens et réduit les inégalités territoriales dans ces pays. Il s'agit donc de l'émergence d'un véritable marché des professionnels de santé.

Emmanuel Bioteau témoigne, rejoint par les représentants du réseau OVR Solidarités, de travaux conduits en Master CDET l'année précédente, et présentés devant OVR Solidarités en amont de ce séminaire, montrant les stratégies d'agences de placement de médecins roumains en France; ces agences suscitent par elles-mêmes la demande (en démarchant des municipalités et des praticiens libéraux proches de la retraite en France) et l'offre (en inondant les praticiens roumains en Roumanie de messages incitants à l'installation professionnelle et familiale en France). Ce faisant, d'agences de placement, elles deviennent de véritables offices de recrutement et de commercialisation.

Le graphique suivant, produit par Despina Vasilcu est suffisamment éloquent: il montre l'accroissement du nombre de médecins roumains en France durant la dernière décennie ; une précision est apportée durant le débat : ce graphique comme l'ensemble de l'analyse ici produite ne prend en compte que les médecins, sans tenir compte de l'ensemble des personnels de santé ! 
Par ailleurs on peut repérer une féminisation croissante des flux migratoires, fait induit à la fois par la féminisation croissante des études en médecine, mais également (cela avait été souligné lors du séminaire de Timisoara en mars 2016) du fait du statut salarial et familial souvent moins favorable pour les femmes dans la société roumaine. Ces dernières seraient donc dorénavant plus enclines à partir.

Cette donnée s'inscrit parmi un ensemble de circonstances qualifiées d' " aggravantes ", en tant que favorables à l'émigration car, en contrepartie, on assiste en Roumanie, dans un contexte général de vieillissement démographique imputable pour partie aux migrations, au vieillissement accéléré du corps médical.

Il vient parmi ces circonstances la dégradation constante du niveau de vie pour l'ensemble de la population roumaine. Les niveaux de revenus des praticiens en Roumanie ne sont pas tant ce qui les pousse à partir semble-t-il que l'équilibre économique précaire qui influe au sens négatif sur le système de santé : moins équipé, moins performant qu'ailleurs. La principale cause de cet état de fait est le sous financement $d u$ système de santé et le manque d'implication des structures gouvernementales dans la résolution des problèmes de santé (fait évoqué préalablement par les témoignages recueillis à Timisoara en mars 2016). L'absence de mesures politiques concrètes ou des politiques incohérentes, sont également pointés par les praticiens roumains migrants comme des causes de leur départ. Au final nombre de médecins témoignent pouvoir ainsi se réaliser professionnellement, être plus à même de bien soigner et donc respecter leurs patients et leur profession à l'étranger. Ils se trouvent ainsi dans la possibilité d'élargir leurs perspectives professionnelles.

Ces derniers évoquent également le ressenti d'un manque de respect et de reconnaissance sociale envers leur profession. Là encore la question de la rémunération ne semble pas être centrale dans le choix de migrer. A conditions de niveaux de vie et de reconnaissance égales, ces médecins indiquent n'être certainement pas partis.

Il reste enfin un dernier argument : la famille. Les conditions de scolarisation et l'avenir personnel des enfants compte énormément dans ces choix.

49 (nb : Les principales conclusions auxquelles aboutit Despina Vasilcu seront remobilisées en conclusion générale de ce séminaire) 
50 Ces témoignages confirment s'il en est l'ensemble des débats tenus jusqu'ici. Les représentants du Comité de jumelage entre Beaupréau (Maine-et-Loire, France) et Posesti (Prahova, Roumanie) rendent compte de leurs projets en faveur d'un développement local coordonné tant en terme d'amélioration des conditions de vie économique qu'en termes d'animation sociale et culturelle. L'absence de personnels de santé est certes problématique, et l'éventualité de la transposition du modèle de maisons de santé est discutée (en somme de la reconstruction de dispensaires mais en y associant des praticiens libéraux en Roumanie). Mais pour l'heure ces initiatives tendent à un constat d'échec.

51 Le problème, souligne Alexandru Dragan, tient dans l'absence d'initiatives. Prolongeant cette réflexion Emmanuel Bioteau lit dans ces résultats l'absence de dynamiques communautaires : les solidarités seront familiales, de voisinages, mais iront rarement au-delà à l'échelle villageoise voire communale. En l'absence de ces ressorts du vivre ensemble, il semble impossible d'envisager quelque évolution de fond en matière de services offerts à la population. Ce faisant les plus jeunes partent, dans les grandes villes ou à l'étranger : pas uniquement pour y trouver un emploi mais également pour y trouver un cadre de vie plus favorable, plus moderne, doté des niveaux d'équipements et de services auxquels ils aspirent. Ces services ne sont cependant pas uniquement d'ordre commercial ; ils peuvent relever, le plus simplement, d'aires de jeux aménagées et entretenues pour les enfants, de la présence d'un dispensaire médical, etc. La cause et la conséquence de ces migrations de personnels médicaux sont donc liées !

\section{Cinq axes de recherches developpées durant ce séminaire}

52 Le champ couvert par les problématiques d'action sociale est large, qui plus est en l'élargissant au secteur médico-social. L'objectif de ce séminaire visait la mise en commun des travaux et de l'expérience des uns et des autres, aux bénéfices de la formation (présence d'étudiants à toutes les rencontres à l'exception de la dernière, laquelle précédait un colloque ouvert aux étudiants), de l'émergence d'un champ de recherche novateur en Roumanie (nous y reviendrons en partie 3 - Perspectives) et d'une valorisation (objectif entre autres de ce compte-rendu). Voici les cinq axes à partir desquels se sont construits les échanges.

\section{(1) Secteur médico-social : sur les effets du changement de système de soins et assurantiel}

53 FLEURET et BIOTEAU écrivaient en 2011: "La santé est un service public parmi d'autres. Mais pour fonctionner de façon optimale, le système de soins nécessite une couverture hospitalière relativement dense et hiérarchisée. L'Etat mène en ce sens un effort de construction. Les communes rurales accueillent des dispensaires à même d'assurer la santé primaire et la prévention au niveau local, ainsi que quelques hôpitaux ruraux aujourd'hui fermés (DUMITRACHE, 2004) ${ }^{5}$. Les villes petites et moyennes disposent de cliniques en capacité de proposer des hébergements de courte durée, et offrant l'accès à la médecine secondaire. Les soins tertiaires sont assurés par un centre hospitalier départemental bâti dans la ville chef-lieu et sous la tutelle duquel sont coordonnées les actions de santé départementales. Les niveaux hiérarchiques 
déterminent le degré de spécialisation des établissements et les volumes d'effectifs alloués en personnels. A cet emboîtement se surimposent des hôpitaux urbains, dont sont dotées les plus grandes villes, qui font office de têtes de réseau des dispensaires de quartiers et, dans les principaux centres universitaires, des cliniques assurant la formation et les soins les plus spécialisés. Des services de santé sont aussi assurés via des dispensaires et hôpitaux sous responsabilités d'entreprises et des grands corps d'Etat (armée, CFR - société nationale des chemins de fer,...) ». Malgré la fin du centralisme d'Etat communiste et jusqu'à la crise économique mondiale de 2008, la santé semble prendre une part croissante dans les priorités gouvernementales en Roumanie : en particulier par un renforcement des allocations budgétaires. Aujourd'hui encore, la réforme du secteur hospitalier, ainsi que celle de la santé mentale, restent inscrites à l'agenda gouvernemental, avec des objectifs identiques de modernisation, d'intégration et de performance. L'enjeu poursuivi est celui de l'efficience budgétaire, faisant dire que la Roumanie est «un État social minimal et un État économique maximal » (ZAMFIR, 1994). A l'instar de tous les PECO, la Roumanie créé, suite à la Révolution de 1989, les conditions de recours à l'offre privée dans de nombreux secteurs d'activités dont les soins et la santé (DURANDIN, 2000). Pour ce seul secteur, la part de l'offre privée passe de l'indice 100 à l'indice 344 entre 1993 et 1999 contre un passage de 100 à 129 pour l'ensemble des services marchands à la population (REY et al., 2000).

54 Les premières mesures législatives visent ainsi à renforcer le secteur de la santé primaire, à améliorer le financement des soins, à décentraliser la décision ou encore à renforcer les compétences managériales des personnels directeurs en santé (par la formation). Le financement des soins par reversions publiques diminue consécutivement à l'introduction d'un système d'assurance santé inspiré du modèle Bismarckien. La réforme du système de santé roumain n'aura été, jusqu'à présent, qu'essentiellement financière. Or, le système de santé roumain est sous financé. Ce problème est accentué par des pertes substantielles, pour certaines imputables à une gestion inadéquate, pour d'autres liées à la corruption. Les causes de ces problèmes sont en général attribuées à des inefficiences structurelles, à des sollicitations du système "non conformes » (examens médicaux non nécessaires, etc.) et à un système qui ne satisfait pas les besoins de la population (LASCU, 2008, 10). La réforme du secteur médico-social n'est donc pas encore aboutie.

\section{(2) Qui pour compenser le retrait de l'État?}

55 L'influence des bailleurs de fonds sur l'État oblige ce dernier à opter pour des politiques auxquelles lui-même comme la population roumaine ne sont pas préparés. Le lien social et les pratiques de solidarité paraissent avoir été durablement reconfigurés par le communisme: l'individualisme l'emporte sur les dynamiques collectives, et les fondements d'une société civile active n'ont pas été reconstitués (MICHEL, 2004). De ce fait, très peu d'initiatives émanent des populations locales, faute de moyens et faute de l'habitude de travailler ensemble, voire par rejet des initiatives collectives.

Le désengagement de l'État, allié à l'absence de relais dans l'économie privée au début des années 1990, constitue le terreau du développement d'initiatives dans l'action sociale émanant d'organisations non gouvernementales (ONG), "souvent avec le soutien de puissants bailleurs de fonds occidentaux (Union européenne, Banque 
Mondiale, grandes ONG internationales) » (PIROTTE, 2009). Ces ONG ont une influence certaine sur les processus de transformation de l'offre de services en appuis aux besoins sociaux de la population dans le pays par leurs actions auprès des plus démunis, par leur relativement bonne couverture territoriale et, non des moindres, par leur poids politique (DRAGAN, 2014).

Quelques grandes organisations internationales sont aujourd'hui incontournables dans le pays à l'instar d'USAID (Agence des USA pour le Développement International), qui en 2006 est en contact avec 20.000 associations sur les 45.000 associations (et fondations) recensées par le Ministère de la Justice de Roumanie (PIROTTE, 2008). Ces ONG tendent à se comporter en locomotives finançant sur projets les associations et initiatives que l'on qualifiera d'émanant de la société civile en cours de formation en Roumanie. C'est pourquoi, suivant HEEMERYCK, «les ONG ont acquis l'avantage, et parfois l'inconvénient pour les États, d'être une des composantes légitimantes et obligatoires de la «bonne " gouvernance; ce qui implique des relations de coopération soutenues entre ces deux types d'acteurs» $(2006,177)$. Néanmoins, plusieurs réserves s'imposent.

Premièrement les budgets des ONG proviennent en grande partie de l'extérieur (BIOTEAU, GLÉMAIN, DRAGAN, 2014). Cette dépendance aux fonds étrangers n'encourage pas les initiatives locales. Plus encore, elle interpelle sur l'autonomie de décision et d'initiative. L'émergence d'une société civile active en Roumanie n'est absolument pas avérée et les actions de ces quelques ONG caritatives sont l'arbre qui cache une forêt qui n'existe pas. "Qu'il existe une vie sociale non étatique est certain (et c'était déjà le cas sous le communisme), qu'une sphère autonome de l'auto organisation politique, syndicale, associative de la société y soit constituée est plus douteux» (COLAS, 2002, 49). Cela tient, comme dit précédemment, à une certaine méfiance envers l'action collective. Et de plus, les ressources internes manquent en Roumanie pour que soient assurées des actions pérennes, principalement en raison de l'absence d'habitude de recours au don dans la société, et faute de capacités d'allocations financières de l'État.

Deuxièmement, le paysage des ONG en Roumanie a été marqué par un mouvement humanitaire international massif dans les années 1990. A cette époque, des informations des plus alarmistes énoncées par les nouvelles autorités sont relayées par les médias occidentaux. Elles paraissent d'autant plus réalistes que l'image d'une population roumaine victime de la misère matérielle et de l'oppression d'un régime ubuesque est déjà largement répandue à l'Ouest depuis au moins le milieu des années quatre-vingt (PIROTTE, 2008). La découverte d'orphelinats insalubres et leur dénonciation par les ONG occidentales ont pérennisé un mouvement d'aide né de l'urgence immédiate après la révolution. Les organisations héritées de cette époque continuent à représenter environ un quart du secteur des ONG en Roumanie. Le problème est que ces organisations ne mobilisent pas localement, n'animent pas la société civile, ne génèrent pas d'initiative collective. Elles sont une nébuleuse de structures étrangères visant à compenser le délitement social post-communiste selon des objectifs particuliers (ciblant les enfants de la rue, les femmes seules, etc.) qui reflètent les préoccupations (et les indignations) des bailleurs de fonds extérieurs, et non les besoins locaux exprimés par la population. 


\section{(3) Structuration du champ de l'économie sociale et solidaire en}

\section{Roumanie}

60 Une thèse de doctorat (géographie, ESO) est actuellement en cours de réalisation sur ce thème à l'Université d'Angers (DRAGAN). Des travaux existent également en Roumanie parmi lesquels, en premier lieu, des études relatives au positionnement des ONG dans le marché des services à la société (RUSU, VILCU et PETRESCU, 2007). Le concept d'économie sociale (Economia Socială) (ES) apparaît pour la première fois en Roumanie, en 2002, dans la Loi HG 829/2002 concernant le Plan national anti-pauvreté et la promotion de l'inclusion sociale. Il est alors associé à un principe qui vise à la fois à développer une société inclusive, et à participer à la construction d'un territoire liant dynamique économique et cohésion sociale. Une nouvelle loi est débattue en 2012 ; le débat stipule pour l'heure que «l'ES représente l'ensemble des activités indépendantes du secteur public, ayant pour objectif de servir l'intérêt général, l'intérêt d'une communauté et/ou des personnes physiques [non juridiques], par l'augmentation de l'occupation des groupes vulnérables et/ou par la production de biens et la prestation des services ", s'inscrivant ainsi en cohérence avec les sept principes énoncés dans la Charte de la Conférence Européenne Permanente des Coopératives, Mutualités, Associations et Fondations (CEP-CMAF). Dans une Europe Sociale qui se cherche à travers ses modèles de protection sociale envisagés à partir de plusieurs typologies développées par TITMUSS (1974) et ESPING-ANDERSEN (1999), auxquelles les recherches engagées dans ce projet référent, et ses appellations: Economie Sociale, Economie Sociale et Solidaire, Tiers-Secteur, Secteur Non Profitable (EVERS et LAVILLE, 2004), la Roumanie n'échappe pas à des représentations plurielles, qu'elles viennent de la population elle-même, des autorités publiques, ou bien des acteurs économiques locaux.

61 Il faut attendre en Roumanie comme ailleurs en Europe la publication des travaux du Johns Hopkins Comparative Non-profit Sector Project (CNP) (1995) et la mise en place en 2001 de la CEP-CMAF. La méthodologie du CNP permet de dérouler une même grille d'analyse malgré les différents contextes étatiques. L'idée consiste à institutionnaliser l'ES à partir d'une comptabilité nationale: en mesurant le poids de ce secteur économique particulier dans la construction et la diffusion d'un compte satellite consacré à l'ES. Il en résulte la volonté de dresser des tableaux convenant à des comparaisons interétatiques, intersectorielles, internationales, voire entre statuts juridiques (les familles) selon les pays concernés et observés. En Roumanie, cette méthodologie comptable et comparative sera appliquée par SAULEAN et EPURE (1998). Ces travaux s'inscrivent plus globalement dans une dynamique de recherche amorcée à la fin des années 1990, portant sur les contributions potentielles de l'ES à la réactivation de dynamiques socioéconomiques de proximité dans les ex PECO. Dans ce contexte CZIKE et KUTI (2006) appréhendent l'ES roumaine dans sa capacité d'intégration sociale à partir de ses organisations charitables. Il s'agit alors d'interroger le rôle des organisations non profitables, souvent étrangères au pays, dans l'émergence et la diffusion d'une action sociale et solidaire en Roumanie. L'ES roumaine est ainsi appréhendée à partir de la production de solidarité sociale en lieu et place d'un État orienté vers le défi de l'efficacité économique dans une Europe encore en construction. Si l'approche macroéconomique peut être intéressante, elle est peu diserte à la fois sur les dynamiques internes aux organisations d'ES (associations et/ou coopératives), et 
sur les organisations qui portent le « modèle " d'ES roumain, à l'instar des travaux de DRAGAN $(2012,2014)$ sur l'ES roumaine ou de GLÉMAIN, BIOTEAU et DRAGAN (2013) sur les finances solidaires en Roumanie. Le remodelage qu'a connu le pays est essentiel pour comprendre à la fois les craintes et les attentes fortes autour de l'économie sociale. Ce contexte interroge les outils à disposition de l'État pour faire face non pas à la crise mais bien aux différentes crises qui l'affectent lui, et sa population. L' Economia Socială saurait-elle relever ce défi de la solidarité et de l'efficacité économique?

\section{(4) Les effets de la transcription des règles communautaires (européenne) sur le terrain}

L'UE intervient dans le financement de la santé en Roumanie de façon indirecte, à travers le programme de pré adhésion PHARE dans un premier temps et, suite à l'adhésion de la Roumanie, par les apports conjoints du Fonds Social Européen (FSE), du Fonds Européen de Développement Régional (FEDER) et du Fonds de Cohésion. Les premières allocations de budgets communautaires ont été destinées à l'amélioration qualitative des dispensaires et à leur équipement, ainsi qu'à la vaccination: cela dès 1991. C'est seulement à partir de 1998 que le Ministère de la Santé publique de Roumanie participe aux programmes de santé publique de l'UE comme la promotion de la santé, la prévention des cancers, la lutte contre le VIH... Les premières influences de l'UE en Roumanie dans le domaine de la santé sont donc du domaine de la santé publique. Au-delà de la santé, l'ensemble des secteurs social et médico-social est concerné par cette transcription des règles communautaires. En raison de son processus d'adhésion à l'Union européenne, la Roumanie a été enjointe d'harmoniser sa législation avec les attendus communautaires (regroupés sous le nom d'Acquis communautaire). Ainsi, le Ministère de la Santé Publique est appelé à ne plus jouer qu'un rôle d'encadrement et de régulation de système. Il doit notamment se désengager totalement de la santé et des soins primaires. Le deuxième impact de l'UE touche donc à la gouvernance et à la gestion des services publics.

Il subsiste une distorsion entre les développements législatifs et leurs applications actuelles sur le terrain, qui résulte d'une faible capacité administrative générale, et au niveau local du manque de clarté dans les responsabilités, ainsi que d'une communication inadéquate entre institutions. Pour exemple, les dispensaires ruraux sont depuis 1998 appelés à être progressivement transformés en l'équivalent de maisons médicales en gestions privées ou publiques. Mais dans les faits, depuis 1998, nombre d'entre eux ont cessé toute activité faute d'investissements privés et de coordination entre municipalités (en charge des locaux), administrateurs (qui par la réforme deviennent gestionnaires managers d'établissements de santé) et personnels de santé (qui passent du statut de salariés à celui de prestataires de services).

\section{(5) Dernier point : les questions relatives à la formation des professionnels et des bénévoles}

De façon transverse, le système de formation roumain est également confronté à ces problématiques de moyens financiers. A partir du milieu des années 1990, les Universités d'État ont commencé à ouvrir des formations à accès payant. Ce dispositif a rapidement conduit à l'ouverture de filières en langues étrangères, en particulier dans 
les spécialités de médecine, mais également dans l'assistance sociale, répondant à des besoins spécifiques pour les étudiants roumains ainsi formés, ces derniers trouvant rapidement par ailleurs des débouchés professionnels à l'étranger. Ce faisant, les filières allophones sont devenues une niche pour les établissements universitaires roumains qui aujourd'hui investissent des moyens financiers et techniques dans la formation d'étudiants étrangers, en plus des ressortissants roumains (POPA, 2014). Si le bénéfice financier immédiat permet de préserver l'équilibre de ces établissements, cette situation accentue encore un peu plus le déséquilibre entre une formation universitaire de professionnels attirés par l'étranger - et des besoins sociaux non résorbés en interne (KRASTEVA et VASILCU, 2014). Les articles de presse généraliste ne manquent pas - tant côté français que côté roumain - sur ce sujet. Sur le plan de la santé, FLEURET et BIOTEAU (2011) y consacrent une partie de leur texte portant sur l'évolution du système de santé et assurantiel en santé roumain. Sur le plan de l'action sociale proprement dite, le séminaire du Centre Ligérien d'Etudes Roumaines du 23 janvier dernier a permis, au travers de la communication de Madame TRANCA (2015), de pointer le risque d'une émigration des jeunes diplômés roumains, en particulier en direction de l'Allemagne et l'Autriche ainsi que du Royaume-Uni. Un récent rapport de l'OCDE (2014) pointe également cette spécificité roumaine: de formations diplômantes internes, soutenues financièrement par l'État, favorisant à la fois une migration entrante de formation à haut niveau de diplomation, et l'émigration des élites. Les conséquences sur les habitants (restant au pays) et sur les territoires : pratiques, flux, mobilités, choix stratégiques des habitants et des collectivités demandent à être étudiés.

\section{Conclusion générale. Perspectives de valorisation et suites données au séminaire}

En conclusion, depuis les années 1990, une large partie du monde, auparavant administrée selon le modèle soviétique, s'est rapidement, et souvent brutalement, ouverte à l'économie de marché et à la pensée néo-libérale. La Roumanie est en première ligne de ce changement. Face à ce constat il apparaît opportun de s'interroger sur les effets sociaux et spatiaux d'un changement de système politique et de ses effets sur l'ensemble des pratiques et des modalités d'action sociale, sanitaire et sociale, assurantiel de santé et d'organisation des soins. Cette rupture qui fait passer d'un modèle de gestion de type collectiviste (le modèle Semashko en santé, la centralisation de toutes décisions et la suppression de 1968 à 1989 du secteur de l'action sociale, s'accompagnant de l'arrêt de la formation et du renvoi au civil des fonctionnaires spécialisés dans le domaine) aux modèles plus libéraux prônés "à l'Ouest " semble aujourd'hui lointaine. Pourtant, la crise, conjoncturelle sur le plan économique, mais en réalité aux fondements structurels, que connaît la Roumanie (et au-delà la majeure partie de l'Europe centrale) remet sur le devant de la scène des questions clefs relatives à la formation des personnels, aux déséquilibres spatiaux dans l'offre de services, et aux effets induits sur la population : émigration, en particulier des jeunes formés en santé et en action sociale, et marchandisation d'un grand nombre de ces services dans un contexte de pénurie des services publics et de ressources financières limitées des habitants usagers. 


\section{BIBLIOGRAPHIE}

Balogh M., 2008, « The role of Romanian NGOs in the democratization process of the society after $1990 »$, in Osborne S.P. (ed.), The Third Sector in Europe. Prospects and challenges , London, Routledge, p. 53-66.

Bioteau E., 2005, Des frontières et des hommes. Approche des recompositions territoriales frontalières : l'exemple de l'Ouest roumain , Thèse de Doctorat de Géographie, Université d'Angers, (28 novembre 2005), $418 \mathrm{p}$. 
Bioteau E., Glemain P., Dragan A., 2014, « L'économie sociale en Roumanie à l'épreuve des représentations et des faits ", in RECMA (Revue internationale de l'économie sociale) , $\mathrm{n}{ }^{\circ} 332$ (avril 2014), p. 46-60.

Cace S. (dir.), 2010, Economia Socială în România - două profiluri regionale [L'économie sociale en Roumanie - deux profils régionaux], Bucarest, Expert.

Colas D., 2002, « L'absence de société civile sous le communisme, sa faiblesse sous le postcommunisme », in D. Colas (dir.), L'Europe post-communiste , Paris, PUF, p. 29-55.

COMISIA PREZIDENTIALA PENTRU ANALIZA SI ELABORAREA POLITICILOR DIN DOMENIUL SANATATII PUBLICE DIN ROMANIA [Commission présidentielle pour l'analyse et l'élaboration des politiques du domaine de la santé publique en Roumanie], 2008, (rapport) « Un sistem sanitar centrat pe nevoile cetateanului » [Un système sanitaire centré sur les besoins des citoyens]. en ligne : http://www.presidency.ro/static/ordine/COMISIASANATATE/ UN_SISTEM_SANITAR_CENTRAT_PE_NEVOILE_CETATEANULUI.pdf

Czike K., Kuti E., 2006, Onketesseg, jotekonysag, tarsadalmi integracio [Volontariat, Charité, Intégration sociale], traduit en anglais par les auteurs, cité dans JENEI G., KUTI E., 2008, « The third sector and civil society ", in OSBORNE S.P. (ed.), The Third Sector in Europe. Prospects and challenges , Londres, Routledge, p. 9-24.

Delépine S., 2007, Quartiers Tsiganes. L'habitat et le logement des Roms de Roumanie en question , L'Harmattan, collection « Aujourd'hui l'Europe », 167 p.

Delépine S., 2006, « Housing of Roma in Central and Eastern Europe : Facts and Proposals », Council of Europe, $14 \mathrm{p}$.

Dobos C., 2006, « Dificultati de acces la serviciile publice de sanatate în România » (Difficultés d'accès aux services publics de santé en Roumanie), in Quality of Life , issue 1-2, p. 7-24.

Dragan A., 2016, Emergence et structuration de l'économie sociale et solidaire en Roumanie, Thèse de doctorat en Géographie, soutenue publiquement le 9 septembre 2016, Université d'Angers et Université de l'Ouest de Timisoara, Angers, $297 \mathrm{p}$.

Dragan A., 2012, Le développement de l'Economie Sociale en Roumanie : quels enjeux et perspectives? L'ESS française, une source potentielle de transfert d'innovation et de futur partenariat, Mémoire de Master 2 Chargé de Développement Entreprises et Territoires durables, Université d'Angers et Observatoire CRESS Pays-de-la-Loire.

Dumitrache L., 2004, Starea de sanatate a populaţiei Romaniei. O abordare geografica [Etat de santé de la population roumaine. Une approche géographique], Editions Univers Enciclopedic, Bucarest, $380 \mathrm{p}$.

Durandin C., 2000, Roumanie, un piège? , Ed. Hesse.

ECSHD, 2006, Romania. Sectorul de Sanatate. Studiu de politica sectoriala [Roumanie. Secteur de la Santé. Etude de politique sectorielle], note du Programme de Développement Humain de la Roumanie, 16 p. [en ligne] : http://siteresources.worldbank.org/INTROMANIAINROMANIAN/ Resources/HealthSectorPolicy NoteRomanian.pdf

Esping-Andersen G., 1999, Social Foundations of Postindustrial Economies , Oxford, Oxford University Press.

Evers A., Laville J-L. (dir.), 2004, The Third Sector in Europe, Cheltenham (UK) \& Northampton (MA-USA), Edward Elgar. 
Fleuret S., Bioteau E., 2011, « Géographie de la santé post-collectiviste en Roumanie : rupture sous influences ", in S. FLEURET \& A-C. HOYEZ (dir.), Santé et géographie. Nouveaux regards, Paris, Economica Anthropos, p.153-177.

Glemain P., Bioteau E., Dragan A., 2013, « La microfinance dans la Roumanie en transition (et en crise). Intérêts et limites d'un secteur émergent, encore peu diffus ", Annals of Public and Cooperative Economics , CIRIEC Canada, vol. 84, issue 2, p. 195-217.

Heemeryck A., 2006, « Gouvernance démocratique, État et ONG en Roumanie : quelques éléments de clarification autour de l'introduction d'une loi de transparence ", in L'Homme et la société, L'Harmattan, 2006/1, n 159 , p. 175-190.

Heemeryck A., 2011, « Les ONG de démocratisation en Roumanie », Multitudes , 4/2011, n 47 , p. $62-70$.

Holt România, 2002, Ghiduri de buna practica în asistenta sociala a copilului si familiei (Guides de bonnes pratiques en assistance sociale de l'enfance et de la famille), Ed. Lumen, Bucarest.

Karabencheva Lévy K., 2010, Politiques publiques à l'égard des minorités ethniques et religieuses après 1989 : étude comparative entre la Roumanie et la Bulgarie, Thèse de doctorat en sciences politiques, ENS Cachan, $545 \mathrm{p}$.

Krasteva A., Vasilcu D., 2014, Migrations en blanc. Médecins d'est en ouest , L'Harmattan.

Lambru M., 2010, Economia socială şi problematica vârstei a treia [L'économie sociale et la problématique du troisième âge], Bucarest, FDSC.

Lambru M., Petrescu C., 2011, Proiect PROMETEUS - Promovarea Economiei Sociale în România prin Cercetare, Educatie si Formare Profesionala la Standarde Europene (Le projet PROMETEUS - Promotion de l'Economie Sociale en Roumanie à travers la Recherche, l'Education et la Formation professionnelle aux standards européens), Document en ligne.

Lascu D., 2008, « Sistemul de asigurari sociale de sanatate din Romania (particularitati la nivelul judetului Tulcea) » [Le système d'assurances sociales de santé en Roumanie (particularités au niveau du département Tulcea)]. Université d'Etat de Moldavie, Chisinau. Manuscrit, Résumé, $27 \mathrm{p}$.

Leroux I., Pujol L., Rigamonti E., 2012, « Nouvelle régulation concurrentielle et nouveaux jeux de proximités. Les associations d'aide à la personne à la reconquête de leur légitimité territoriale », in Revue d'Economie Régionale et Urbaine, $\mathrm{n}^{\circ}$ 3, p. 407-430.

Michalon B., 2013, « Mobility and Power in Detention. The Management of Internal Movement and Governmental Mobility in Romania » in D.MORAN \& D.COLON, Carceral Geographies: Mobility and Agency in Emprisonment and Migrant Detention, Ashgate.

Michel P. (dir.), 2004, Europe centrale, la mélancolie du réel ,CERI/Autrement, Paris, 141 p.

OCDE, 2014, Perspectives des migrations internationales 2014. Roumanie , en ligne : 10.1787/ migr_outlook-2014-fr.

Pirotte G., 2008, « La société civile roumaine post-communiste », Autrepart , (48), p. 153-164.

Pirotte G., 2009, « Engagement citoyen et société civile », Sociologies [en ligne], Transferts. L'engagement citoyen en Europe centrale et orientale, [mis en ligne le 05 novembre 2009] : http://sociologies.revues.org/index3017.html

Pirotte G., 2010, «La notion de société civile dans les politiques et pratiques du développement : Succès et ambiguïtés d'un concept en perpétuelle gestation ", Revue de la régulation. Capitalisme, 
institutions, pouvoirs , $\mathrm{n}^{\circ} 7,1^{\mathrm{er}}$ semestre 2010, mis en ligne le 03 juin 2010, [en ligne] : http:// regulation.revues.org/7787.

Popa N., Luches D., 2014, «Etudier la médecine pour émigrer ? ", in A. Krasteva et D. Vasilcu, Migrations en blanc. Médecins d'est en ouest, L'Harmattan, p. 83-100.

Rey V. (coord.), Groza O., Ianos I., Patroescu M., et al., 2000, (2007 éd. aug.), Atlas de la Roumanie , CNRS-Libergéo - La Documentation française, collection Dynamiques du territoire, RECLUS, $167 \mathrm{p}$.

Robin R., 2011, « Former à la santé publique par l'étude de milieu », Revue Soins , n 760, novembre 2011

Robin R., 2014, « Accompagner sans - trop - normaliser ? », in Revue Actualités Sociales Hebdomadaires, $\mathrm{n}^{\circ} 2884 \mathrm{du} 21 / 11 / 2014$.

Roth M., 1999, « Children's Rights in Romania: Problems and Progress », in Social Work in Europe, Volume 6, Number 3, p.30-37.

Rusu O., Vilcu I., Petrescu C., 2007 (mai), Locul si Rolul Organizatiilor Neguvernamentale pe Piata de Servicii Sociale din Romania (Place et Rôle des ONG dans le Marché des Services Sociaux en Roumanie), Fundatia pentru Dezvoltarea Societatii Civile.

Saulean D., Epure C., 1998, Defining the Nonprofit Sector: Romania, The Johns Hopkins Comparative Nonprofit Sector, John Hopkins University Press, Project Series, $\mathrm{n}^{\circ} 32$.

Scutaru B., 2013, Les relations entre les sociétés française et roumaine des années 1960 à 1995 : un atout pour l'ancrage de la Roumanie à l'Europe ?, Thèse de doctorat en Histoire, Université d'Angers.

Scutaru B., 2014, « Local practices of humanitarian aid: Pharmaciens sans frontières Anjou in Romania during the 1990s », Eastern Journal of Eruopean Studies, Volume 5, issue 2, p. 61-75.

Titmuss R.M., 1974, Social Policy: an Introduction, Melbourne, Allen \& Unwin.

Vladescu C., Scintee G., Olsavszky V., 2008, Romania Health system review , [ Health System in Transition, Vol. 10, $\mathrm{N}^{\circ}$ 3, 2008, S. Allin \& P. Mladovsky (ed.)], European Observatory on Health Systems and Policies, $206 \mathrm{p}$.

Vlasceanu M., 1996, Sectorul non-profit. Contexte, organizare, conducere [Le secteur non-profit. Contextes, organisations, conduites], Bucarest, Panaidea.

Zamfir E., 1995, « Politica de protectie a copilului in Romania » (Protection de l'Enfance en Roumanie), in C. Zamfir, \& E. Zamfir (coord.), Politici sociale in context european (Politiques sociales dans le contexte européen), Polirom, Bucarest.

Zamfir C., 1996, « La politique sociale dans la Roumanie en transition : hypothèses concernant l'explication d'un paradoxe ", in N. Pelissier, A. Marrié et F. Despres, La Roumanie contemporaine. Approches de la «transition », Paris, L’Harmattan, p. 179-194.

\section{NOTES}

1. Outre la finalisation de ce cycle de 3 rencontres (Angers, séminaire du Centre Ligérien d'Etudes Roumaines du 12 juin 2015 ; Timisoara, séminaire du 24 mars 2016), ce séminaire a également constitué un temps inaugural du colloque "L'Economie Sociale et Solidaire dans les Politiques publiques territoriales. Regards croisés acteurs, élus, chercheurs » (7, 8 et 9 juin 2016 à Angers). Les participants au séminaire (invités) 
ont également été conviés à communiquer durant ce colloque, lequel consacre un atelier aux problématiques de santé en Roumanie et élargit le périmètre spatial du séminaire par des ateliers consacrés aux problématiques de développement social et solidaire en Europe centrale et orientale (Bulgarie, Hongrie, Serbie en plus de la Roumanie).

2. Les mortalités maternelle et infantile enregistrées au milieu des années 1990 sont revenues à leur niveau des années 1970 (ECSHD, 2006, 2).

3. INS, 2015.

4. http://www.dsptimis.ro/programe-nationale-de-sanatate/programul-national-deprevenire-a-sarcinilor-nedorite-12-12-2014 - 27.08.2016

5. Précisons que les communes roumaines sont vastes (moyenne théorique de 8.500 ha, pour environ 3.300 habitants). Elles ont connu un processus de concentration dans les décennies 1950 et 1960 (processus aboutit et stabilisé par la réforme territoriale de 1968). Le regroupement de plusieurs villages (jusqu'à 20-40 dans les communes des Carpates) en une seule entité administrative communale a pour principale conséquence le renforcement des centres de ces communes, lesquels ont été progressivement pourvus en équipements et services de base. Les villages satellites souffrent de leur côté de moins bonnes dessertes routières (si route il $\mathrm{y} a$ !), et subséquemment en période socialiste de l'absence de réseaux d'adduction d'eau, gaz, électricité, téléphone, etc. (ces défauts d'infrastructures persistent aujourd'hui fréquemment).

\section{RÉSUMÉS}

Cet atelier conclut un cycle de 3 rencontres organisées en 2015 et en 2016, portant sur les problématiques du secteur médico-social en Roumanie, regroupées sous l'intitulé « Emergence et structuration d'un secteur médico-social en Roumanie ». Il s'agit ici de produire (1) une typologie des différentes situations, et l'analyse de ces situations, (2) de comprendre les effets des régulations publiques à caractères social et médical sur les territoires, leurs habitants, leurs établissements, (3) d'analyser les raisons du non-recours à certains services et (4) de formaliser les modalités (leur nécessité réelle ?, leur applicabilité ?) d'un transfert de pratiques entre la France et la Roumanie (et inversement) tant en termes de formation que pour l'action publique et/ou civile locale ${ }^{1}$.

This workshop concludes a cycle of 3 meetings organized in 2015 and in 2016. It relates to the problems of both the medical sector and the social sector in Romania. Entitled "Emergence and structuring of a medical and social sector in Romania", the workshop and all the former associated meetings aim at: (1) Establishing a typology of the various situations, and analysing these situations; (2) Understanding the effects of the public regulations(with social and medical dimensions) on places, their inhabitants, their establishments; (3) Analysing the reasons of the non-use of certain services; (4) Formalising the conditions of a transfer of skills and practices between France and Romania both in terms of training and of actions for the local public and/or civil. 
INDEX

Mots-clés : régulation publique, déséquilibres territoriaux, non-recours, transferts de pratiques Index géographique : Roumanie

\section{AUTEUR}

EMMANUEL BIOTEAU

Laboratoire ESO-Angers (UMR 6590 CNRS) 